\title{
CARTESIAN-CLOSED COREFLECTIVE SUBCATEGORIES OF UNIFORM SPACES
}

\author{
BY
}

\author{
M. D. RICE AND G. J. TASHJIAN ${ }^{1}$
}

\begin{abstract}
This paper characterizes the coreflective subcategories $e$ of uniform spaces for which a natural function space structure generates the exponential law $X^{Y \otimes Z}=\left(X^{Y}\right)^{Z}$ on $\mathcal{C}$. Such categories are cartesian-closed. Specifically, we show that $\mathcal{C}$ is cartesian-closed in this way if and only if $\mathcal{C}$ is inductively generated by a finitely productive family of locally fine spaces. The results divide naturally into two cases: those subcategories containing the unit interval are generated by precompact spaces, while the subcategories not containing the unit interval are generated by spaces which admit an infinite cardinal. These results may be used to derive the characterizations of cartesian-closed coreflective subcategories of Tychonoff spaces found in [10].
\end{abstract}

Fundamentals. Unif will denote the category of Hausdorff uniform spaces and uniformly continuous mappings. All subcategories of Unif are assumed to be full, isomorphism-closed, and to contain a nonempty space.

Definition. A category $\mathcal{C}$ with finite products $X \oplus Y$ is cartesian-closed if for each $X$ in $\mathcal{C}$, the functor $X \otimes-: \mathcal{C} \rightarrow \mathcal{C}$ has a right adjoint.

The action of the right adjoint to $X \otimes$ - will be denoted by $Y \rightarrow[X, Y]$, for $Y$ in $\circlearrowright$. The values $[X, Y]$ are called exponential objects and they satisfy the hom-set condition

$$
\mathcal{C}(Z \otimes X, Y)=e(Z,[X, Y])
$$

for $X, Y$ and $Z$ in $\mathcal{C}$, where equality represents a bijection which is natural in $Z$ and $Y$. Furthermore, $\bigodot$ satisfies the exponential law

$$
[Z \otimes X, Y]=[Z,[X, Y]],
$$

where equality represents a $\mathcal{C}$-isomorphism. (The reader is referred to [7] for further information.)

Now assume that $C$ is a coreflective subcategory of Unif and let $c:$ Unif $\rightarrow \subseteq$ be the coreflection operator which is right adjoint to the inclusion operator $\mathcal{C} \rightarrow$ Unif. We mention the following basic facts found in [6]. A subfamily $C$ of Unif is coreflective if and only if $C$ is closed under the formation of uniform sums and quotients. The coreflection $c X$ of any space $X$ may be assumed to have the same

Received by the editors August 8, 1978 and, in revised form, March 3, 1982.

1980 Mathematics Subject Classification. Primary 54E15; Secondary 18D15.

${ }^{1}$ Written while the second author was a National Academy of Sciences Exchange Scientist in Czechoslovakia, 1977-1978. 
underlying set, with the identity mapping $c X \rightarrow X$ uniformly continuous and satisfying the following universal property: if $f: C \rightarrow X$ is uniformly continuous ( $C$ in $C$ ), then $f: C \rightarrow c X$ is uniformly continuous. Note that each coreflective subcategory $C$ of Unif has finite products with the definition $X \otimes Y=c(X \times Y)$, where $X \times Y$ denotes the usual uniform product.

Let $\delta$ be a nonempty subfamily of Unif. Then $\operatorname{co}(\mathcal{S})$ will denote the coreflective hull of $\delta$ : the smallest coreflective subcategory of Unif containing $\delta$, which consists of all quotients of sums of members from $\mathcal{S}$. If $\mathcal{C}=\operatorname{co}(\mathcal{S})$, we say that $\mathcal{S}$ inductively generates $\mathcal{C}$.

By the results found in [4], a coreflective subcategory of Unif is cartesian-closed if and only if the product $\otimes$ preserves sums and quotients. The product $X$ in Unif preserves quotients (see [5, III, Exercise 8]), but it does not preserve sums (for example, $[0,1] \times \mathbf{N}$ is not the uniform sum of countably many copies of $[0,1])$; hence Unif is not cartesian-closed.

The following notation will be used throughout the paper. For $X$ and $Y$ in Unif, $U(X, Y)$ denotes the family of uniformly continuous mappings from $X$ into $Y .|A|$ denotes the cardinality of the set $A$.

Proposition 0. Let $\mathcal{C}$ be a cartesian-closed subcategory of Unif which contains the one-point space $T$. Then $|[X, Y]|=|U(X, Y)|$ for all $X$ and $Y$ in $\mathcal{C}$.

Proof. Since $T \otimes X$ is isomorphic to $X$, one obtains $|U(X, Y)|=|U(T \otimes X, Y)|$ $=|U(T,[X, Y])|=|[X, Y]|$.

The above proposition shows that for any cartesian-closed coreflective subcategory $\mathcal{C}$ of Unif, we may assume that $[X, Y]$ has the underlying set $U(X, Y)$ without affecting the naturality of the mappings. Hence we can give the following reformulation of the original definition:

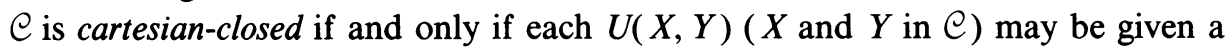
uniform structure such that

(i) $U(X, Y)$ is a member of $\mathcal{C}$,

(ii) the evaluation mapping $e: U(X, Y) \otimes X \rightarrow Y$ defined by $e(f, x)=f(x)$ is uniformly continuous, and

(iii) for each $Z$ in $\bigodot$ and uniformly continuous mapping $h: Z \otimes X \rightarrow Y$, the mapping $\hat{h}: Z \rightarrow U(X, Y)$ defined by $\hat{h}(z)(x)=h(z, x)$ is uniformly continuous.

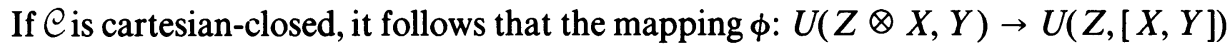
defined by $\phi(h)=\hat{h}$ is a natural bijection and induces a uniform isomorphism of $[Z \otimes X, Y]$ and $[Z,[X, Y]]$.

The preceding statements may be used to establish the following proposition (essentially found in Lemma 1 of [8 and 10]).

Proposition 1. Assume $\mathcal{S} \subset$ Unif and $\mathcal{C}=\operatorname{co}(\mathcal{S})$. Assume that for each $S$ in $\mathcal{S}$ the functor $S \otimes-: \mathcal{C} \rightarrow \mathcal{C}$ has a right adjoint $Y \rightarrow[S, Y]$. Then $\mathcal{C}$ is cartesian-closed and $[X, Y]$ is projectively generated in $C$ by the family of mappings $\{[f]:[X, Y] \rightarrow[S, Y]\}$, where $S$ is in $\mathcal{S}, f: S \rightarrow X$ is uniformly continuous, and $[f]$ is defined by $[f](g)=g \circ f$. 
A uniformity on a set $X$ may be described in terms of covers, pseudometrics, or neighborhoods of the diagonal, respectively. The reader is referred to [5] for the basic axioms and definitions.

If $\mathscr{Q}$ is a cover of the set $X$ and $A \subset X$, define $\operatorname{St}(A, \mathcal{Q})=\cup\{U \in \mathscr{U}: U \cap A \neq$ $\varnothing\}$ and $\mathscr{Q}^{*}=\{\operatorname{St}(x, \mathcal{Q}): x \in X\}$. The following function space uniformities on $U(X, Y)$ will be used in the sequel:

(1) The uniformity of uniform convergence on $U(X, Y)$ has the base consisting of the covers $\hat{U}$ induced by the uniform covers $\mathcal{Q}$ of $Y$ in the following manner: For $f \in U(X, Y)$, define $U_{f}=\{g \in U(X, Y): g(x) \in \operatorname{St}(f(x)$, Q ) for all $x$ in $X\}$ and set $\hat{\mathcal{U}}=\left\{U_{f}: f \in U(X, Y)\right\}$. The notation $U(X, Y)$ will imply that the uniformity under consideration is the uniformity of uniform convergence.

(2) Let $\delta$ be a family of uniform spaces. The function space uniformity on $U(X, Y)$ which is projectively generated by the mappings $[f]: U(X, Y) \rightarrow U(S, Y)$, where $S$ is a member of $\mathcal{S}$, and $f: S \rightarrow X$ is uniformly continuous, and each $U(S, Y)$ has the uniformity of uniform convergence, will be denoted by $U_{\S}(X, Y)$.

In general, the identity mapping $U(X, Y) \rightarrow U_{\delta}(X, Y)$ is uniformly continuous. If the family $\delta$ is closed under the formation of uniformly continuous images, $U_{\delta}(X, Y)$ may be described as the uniformity of uniform convergence on the subspaces of $X$ which are uniformly isomorphic to members of $\mathcal{S}$.

Our next result follows from the preceding definitions and Proposition 1.

Corollary 1. Assume $\delta \subset$ Unif and $\mathcal{C}=\operatorname{co}(\delta)$ with the coreflection operator $c$ : Unif $\rightarrow \mathcal{C}$. Assume that for each $S$ in $\delta$ the functor $S \otimes-: \mathcal{C} \rightarrow \mathcal{C}$ has a right adjoint

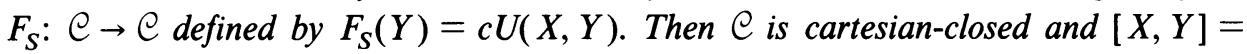
$c U_{\S}(X, Y)$ for all $X$ and $Y$ in $\bigodot$.

For example, let $\delta$ be either the class of compact spaces or the class of precompact spaces (the subspaces of compact spaces). Then $\operatorname{co}(\mathscr{S})$ is cartesian-closed and $[X, Y]=c U_{\S}(X, Y)$ for all $X$ and $Y$ in $\mathcal{C}$ (see [9] or Theorem 1 below).

The main purpose of the present paper is to characterize the subfamilies $\delta$ of Unif such that $\mathcal{C}=\operatorname{co}(\delta)$ is cartesian- closed with the exponential $[-,-]=c U_{\delta}(-,-)$, where $c$ denotes the coreflection operator.

Convention. Throughout this paper $\mathcal{S}, \mathcal{C}$, and $c$ will have the meanings given above. If $f: A \times B \rightarrow C$ is a mapping, $\hat{f}$ will denote the mapping defined by $\hat{f}(a)(b)=f(a, b)$. If $f: A \rightarrow B$ is a uniformly continuous mapping, [f]: $U(B, C) \rightarrow$ $U(A, C)$ will denote the mapping defined by $[f](g)=g \circ f$. Finally, $e: U(X, Y) \times$ $X \rightarrow Y$ denotes the evaluation mapping defined by $e(f, x)=f(x)$.

Cartesian-closed coreflective hulls. For $X$ and $Y$ in Unif, the semi-uniform product $X * Y$ of $X$ and $Y$ is the uniform space projectively generated by the mappings $f$ : $X \times Y \rightarrow Z, Z$ in Unif, such that the family $\left\{f_{y}=f(-, y): X \rightarrow Z\right\}$ is equiuniformly continuous and each mapping $f_{x}=f(x,-): Y \rightarrow Z$ is uniformly continuous. The operation $*$ is associative and noncommutative (for example, $\mathbf{N} *[0,1] \neq$ $[0,1] * \mathbf{N})$ and $U(X * Y, Z)=U(X, U(Y, Z))$, where the equality denotes a uniform isomorphism (see [5] for details). In addition, one may establish that a mapping $f$ : $X \times Y \rightarrow Z$ is uniformly continuous if and only if $f: X * Y \rightarrow Z$ is uniformly continuous and $f^{\prime}: Y * X \rightarrow Z$ is uniformly continuous, where $f^{\prime}(y, x)=f(x, y)$. 
Definition 1. $\delta$ satisfies Axiom $I$ if $S \times T \in \mathcal{C}$ for all $S$ and $T$ in $\mathcal{S}$.

Proposition 2. The following statements are equivalent:

(1) s satisfies Axiom I.

(2) The identity mapping $X * S \rightarrow X \otimes S$ is uniformly continuous for all $S$ in $\delta$ and $X$ in $\mathrm{C}$.

If $\mathcal{C}$ is cartesian-closed, then (1) and (2) are equivalent to the following statements:

(3) The identity mapping $[S, Y] \rightarrow c U(S, Y)$ is uniformly continuous for all $S$ in $\mathcal{S}$ and $Y$ in $\mathrm{C}$.

(4) The identity mapping $[X, Y] \rightarrow c U_{\S}(X, Y)$ is uniformly continuous for all $X$ and

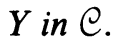

Proof. (2) $\rightarrow$ (1). Let $S$ and $T$ be members of $\mathcal{\delta}$. By (2) the identity mappings $S * T \rightarrow S \otimes T$ and $T * S \rightarrow T \otimes S$ are uniformly continuous. Since the operation $\otimes$ is commutative, it follows from the preceding comments that $S \times T \rightarrow S \otimes T$ is uniformly continuous, so $S \times T \in \mathcal{C}$.

$(1) \rightarrow(2)$. Choose a quotient mapping $q: \Sigma S_{i} \rightarrow X$, where $\Sigma S_{i}$ is a sum of members from $\delta$. Define $q_{i}=\left.q\right|_{S_{i}}$ and let $j: T \rightarrow T$ be the identity mapping for $T$ a member of $\mathcal{S}$. By (1), $r_{i}=q_{i} \times j: S_{i} \times T \rightarrow X \otimes T$ is uniformly continuous. Let $\hat{r}_{i}: S_{i} \rightarrow$ $U(T, X \otimes T)$ be the mapping associated with $r_{i}$ and let $\hat{k}: X \rightarrow U(T, X \otimes T)$ be the mapping defined by $\hat{k}(x)(t)=(x, t)$. One easily verifies that $\hat{k} \circ q=\Sigma \hat{r}_{i}$; since $q$ is a quotient mapping it follows that $\hat{k}$ is uniformly continuous, so the identity mapping $X * T \rightarrow X \otimes T$ is uniformly continuous.

$(4) \rightarrow(3)$ is immediate and (1) $\rightarrow(3)$ is Corollary 2 in [9].

(3) $\rightarrow$ (2). Define $Y=X \otimes S$ and let $i: X \otimes S \rightarrow Y$ be the identity mapping. Since $\circlearrowright$ is cartesian-closed, $\hat{i}: X \rightarrow[S, Y]$ is uniformly continuous; hence, by (3), $\hat{i}: X \rightarrow c U(S, Y)$ is uniformly continuous, so $i: X * S \rightarrow Y$ is uniformly continuous.

(3) $\rightarrow$ (4). If $S$ is a member of $\delta$ and $f: S \rightarrow X$ is uniformly continuous, the induced mapping $[f]:[X, Y] \rightarrow[S, Y] \rightarrow U(S, Y)$ is uniformly continuous by (3). Since $U_{\S}(X, Y)$ is projectively generated by all mappings of the form [ $\left.f\right]$, it follows that $[X, Y] \rightarrow c U_{\delta}(X, Y)$ is uniformly continuous.

DEFINITION 2. $\delta$ satisfies Axiom $I I$ if for each uniformly continuous mapping $\hat{f}: S \rightarrow U(S, Y)(S$ in $\delta$ and $Y$ in $\mathcal{C})$, the diagonalized mapping $f_{\Delta}: S \rightarrow Y$ defined by $f_{\Delta}(s)=\hat{f}(s)(s)$ is unformly continuous.

PROPOSITION 3. The following statments are equivalent:

(1) ऽ satisfies Axiom II.

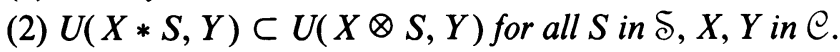

(3) The evaluation mapping $e: c U(S, Y) \otimes S \rightarrow Y$ is uniformly continuous for all $S$ in $\delta$ and $Y$ in $\mathrm{C}$.

(4) The evaluation mapping $e: c U_{\S}(X, Y) \otimes X \rightarrow Y$ is uniformly continuous for all $X$ and $Y$ in $\bigodot$.

If $\mathcal{C}$ is cartesian-closed, then (1)-(4) are equivalent to the following statements:

(5) The identity mapping $c U(S, Y) \rightarrow[S, Y]$ is uniformly continuous for all $S$ in $\mathcal{S}$ and $Y$ in $\mathrm{C}$. 
(6) The identity mapping $c U_{\S}(X, Y) \rightarrow[X, Y]$ is uniformly continuous for all $X$ and $Y$ in $\mathrm{C}$.

(7) $U(X * Y, Z) \subset U(X \otimes Y, Z)$ for all $X, Y$ and $Z$ in $\bigodot$.

Proof. (4) $\rightarrow(3)$ is immediate.

(3) $\rightarrow$ (2). If $f \in U(X * S, Y)$, the associated mapping $\hat{f}: X \rightarrow c U(S, Y)$, and hence the product mapping $\hat{f} \times i: X \otimes S \rightarrow c U(S, Y) \otimes S$, are uniformly continuous, where $i: S \rightarrow S$ is the identity mapping. Hence by (3), $f=e \circ(\hat{f} \times i): X \otimes S \rightarrow Y$ is uniformly continuous.

$(2) \rightarrow(1)$. If $\hat{f}: S \rightarrow U(S, Y)$ is uniformly continuous, the associated mapping $f: S * S \rightarrow Y$ is uniformly continuous, so by (2), $f \in U(S \otimes S, Y)$. Since the diagonal mapping $\Delta: S \rightarrow S \otimes S$ is uniformly continuous, it follows that $f \circ \Delta=f_{\Delta} \in$ $U(S, Y)$; hence Axiom II is satisfied.

$(1) \rightarrow(4)$. Since $e=\operatorname{co}(\mathcal{S})$, it suffices to show that $e \circ q: S \rightarrow Y$ is uniformly continuous for each uniformly continuous mapping $q: S \rightarrow c U_{\S}(X, Y) \times X(S$ in $\delta)$. Define $f=\pi_{2} \circ q: S \rightarrow X$ and $g=\pi_{1} \circ q: S \rightarrow U_{\delta}(X, Y)$, where $\pi_{1}$ and $\pi_{2}$ are projection mappings. By definition, the mapping $[f]: U_{\delta}(X, Y) \rightarrow U(S, Y)$ is uniformly continuous. Define $\hat{h}=[f] \circ g: S \rightarrow U(S, Y)$. By Axiom II, the mapping $h_{\Delta}$ is uniformly continuous and one easily verifies that $h_{\Delta}=e \circ q$.

If $C$ is cartesian-closed, $[X, Y]$ carries the smallest uniformity on $U(X, Y)$ such that $e:[X, Y] \otimes X \rightarrow Y$ is uniformly continuous. Hence (6) $\rightarrow(4)$. The implications $(3) \rightarrow(5)$ and $(7) \rightarrow(2)$ are evident, while the proof of $(2) \rightarrow(1)$ given above also establishes $(5) \rightarrow(1)$.

Finally, to establish (6) $\rightarrow$ (7), assume that $f \in U(X * Y, Z)$. By (6), the associated mapping $\hat{f}: X \rightarrow c U(Y, Z) \rightarrow c U_{\S}(Y, Z) \rightarrow[Y, Z]$ is uniformly continuous; hence $f: X \otimes Y \rightarrow Z$ is uniformly continuous.

DEFINITION 3. $\delta$ satisfies Axiom $I I I$ if, for each pair of uniformly continuous mappings $\hat{f}: R \rightarrow U(S, Y)$ and $g: T \rightarrow S(R, S, T$ in $\mathcal{S}$ and $Y$ in $\mathcal{C})$, the family $\hat{f}[R] \circ g=\{\hat{f}(r) \circ g: T \rightarrow Y\}$ is equi-uniformly continuous.

RemarK. We note that if $\delta$ satisfies Axiom III, then it also satisfies Axiom II. (Let $R=S=T$ and let $g: S \rightarrow S$ be the identity mapping. By Axiom III, $\hat{f}: S \rightarrow U(S, Y)$ uniformly continuous implies that $\hat{f}[S]$ is equi-uniformly continuous; hence the associated mapping $f: S \times S \rightarrow Y$ is uniformly continuous, so $f_{\Delta}=f \circ \Delta: S \rightarrow Y$ is uniformly continuous.)

PROPOSITION 4. The following statements are equivalent:

(1) $\delta$ satisfies Axiom III.

(2) $U(S * T, Y) \subset U(S \times T, Y)$ for all $S, T$ in $\delta$ and $Y$ in $\mathrm{C}$.

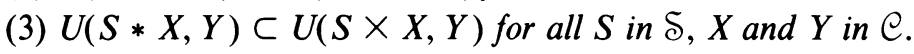

Proof. (3) $\rightarrow(2)$ is immediate.

$(1) \rightarrow(2)$. Choose $f \in U(S * T, Y)$. Then the associated mapping $\hat{f}: S \rightarrow U(T, Y)$ is uniformly continuous, so by Axiom III (with $g: T \rightarrow T$ the identity mapping), $\hat{f}[S]$ 
is equi-uniformly continuous. Hence $f \in U(S \times T, Y)$ based on the remarks at the beginning of this section.

(2) $\rightarrow(1)$. Let $\hat{f}: R \rightarrow U(S, Y)$ and $g: T \rightarrow S$ be uniformly continuous mappings. Then the associated mapping $f: R * S \rightarrow Y$ is uniformly continuous, so by (2) $f \in U(R \times S, Y)$. Let $i: R \rightarrow R$ be the identity mapping. Then $h=f \circ(i \times g)$ : $R \times T \rightarrow R \times S \rightarrow Y$ is uniformly continuous, so the family $\left\{h_{r}=h(r,-): T \rightarrow Y\right\}$ is equi-uniformly continuous and one easily verifies that $h_{r}=\hat{f}(r) \circ g$.

(2) $\rightarrow(3)$. Let $f \in U(S * X, Y)$, let $q: \Sigma T_{i} \rightarrow X$ be a quotient mapping where $\left\{T_{i}\right\}$ is a family of members from $\mathcal{S}$, and let $i: S \rightarrow S$ be the identity mapping. By [5, Exercise III.8] $i \times q: S \times \Sigma T_{i} \rightarrow S \times X$ is a quotient mapping. Since $f \circ(i \times q)$ : $S * \Sigma T_{i} \rightarrow S * X \rightarrow Y$ is uniformly continuous, the restriction $r_{i}$ of $f \circ(i \times q)$ to $S * T_{i}$ is uniformly continuous, so by (2) $r_{i} \in U\left(S \times T_{i}, Y\right)$. Hence for each $i$, $\left\{\left(r_{i}\right)_{s}=r_{i}(s,-): T_{i} \rightarrow Y\right\}$ is an equi-uniformly continuous family. It follows that the family $\left\{(f \circ(i \times q))_{s}=f \circ(i \times q)(s,-): \Sigma T_{i} \rightarrow Y\right\}$ is also equi-uniformly continuous. Therefore the mapping $f \circ(i \times q): S \times \Sigma T_{i} \rightarrow Y$ is uniformly continuous. Since $i \times q$ is a quotient mapping, it follows that $f \in U(S \times X, Y)$.

THEOREM 1. The following statements are equivalent:

(1) $\mathcal{C}$ is cartesian-closed and $[S, Y]=c U(S, Y)$ for all $S$ in $\mathcal{S}$ and $Y$ in $\mathcal{C}$.

(2) $\mathcal{C}$ is cartesian-closed and $[X, Y]=c U_{\delta}(X, Y)$ for all $X$ and $Y$ in $\bigodot$.

(3) S satisfies Axioms I and II.

(4) $\mathcal{S}$ satisfies Axioms I and III.

(5) $U(S * T, Y)=U(S \otimes T, Y)$ for all $S, T$ in $\delta, Y$ in $\bigodot$.

Proof. (4) $\rightarrow$ (3) follows from the remark preceding Proposition 4.

(2) $\rightarrow(1)$ is immediate from the definition of $U_{\delta}(X, Y)$.

(5) $\rightarrow$ (4). By (5), the identity mapping $S * T \rightarrow S \otimes T$ is uniformly continuous. Then the proof of $(2) \rightarrow(1)$ in Proposition 2 shows that $\delta$ satisfies Axiom I. By (5) and Axiom I, the Proposition 4 implies that $\delta$ satisfies Axiom III, so (4) holds.

(1) $\rightarrow$ (5). By Proposition 3, $U(S * T, Y) \subset U(S \otimes T, Y)$ and by Proposition 2, $S * T \rightarrow S \otimes T$ is uniformly continuous, so (5) holds.

(3) $\rightarrow$ (2). By Propositions 2 and 3, $U(X \otimes S, Y)=U(X * S, Y)=U(X, U(S, Y))$

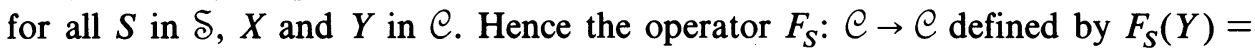
$c U(S, Y)$ is right adjoint to the functor $S \otimes-: \bigodot \rightarrow \bigodot$, so by Proposition $1, \mathcal{C}$ is cartesian-closed and $[X, Y]=c U_{\S}(X, Y)$.

Remark. Assume that $\delta$ satisfies the conditions in Theorem 1. If $X=\Sigma S_{i}$ is the sum of members from $\delta$, then $[X, Y]=c U_{\S}(X, Y)$ is the coreflection of the uniformity of the uniform convergence on the summands of $X$. To prove this fact, first observe that the identity mapping $[X, Y]=c U_{\S}(X, Y) \rightarrow c U_{\hat{\delta}}(X, Y)$ is uniformly continuous, where $U_{\hat{\delta}}(X, Y)$ denotes the uniformity described above. By a result in $[4],[X, Y]$ is naturally isomorphic to $c \Pi\left[S_{i}, Y\right]$ via the mapping $e$, where $e(f)=\left(\left.f\right|_{S_{i}}\right)$ (the restriction mapping in each coordinate). Since $\left[S_{i}, Y\right]=c U\left(S_{i}, Y\right)$ and the restriction mapping $U_{\hat{\delta}}(X, Y) \rightarrow U\left(S_{i}, Y\right)$ is uniformly continuous, it follows 
that the identity mapping $c U_{\hat{\delta}}(X, Y) \stackrel{e}{\rightarrow} c \Pi U\left(S_{i}, Y\right) \stackrel{e^{-1}}{\rightarrow}[X, Y]$ is uniformly continuous, which concludes the proof.

Locally fine spaces. Some significant equivalent statements may be added to those in Theorem 1 using ideas connected with locally fine spaces.

Recall that a uniform space is discrete if the cover of $X$ by singleton sets is uniform. $D$ will denote the collection of all discrete uniform spaces. We remark that $\mathscr{D}$ is the smallest coreflective subcategory of Unif and $\mathscr{D}$ is cartesian-closed since it is isomorphic to the category of sets and mappings.

A uniform space $X$ is locally fine if for each uniform cover $\left\{U_{i}\right\}$ and family of uniform covers $\mathscr{V}_{i}, i \in I$, the cover $\left\{U_{i} \cap V: V \in \mathscr{V}_{i}, i \in I\right\}$ is uniform. $\Lambda$ will denote the collection of all locally fine spaces, which forms a coreflective subcategory of Unif (see [2]). The primary examples of locally fine spaces are the familiar precompact spaces and fine spaces. It has recently been shown by J. Pelant that the locally fine spaces are precisely the subspaces of the fine spaces.

Let $m$ be an infinite cardinal. A uniform space $X$ has covering character $m$ if $m$ is the smallest cardinal such that $X$ has a base consisting of uniform covers, each of power less than $m$ (equivalently, $m$ is the smallest cardinal such that $X$ has no uniformly discrete subspace of power $m) . \mathcal{C}(m)$ will denote the class of all uniform spaces having covering character $m$.

A uniform space $X$ admits $m$ if for each family $\left\{\mathscr{Q}_{i}\right\}, i \in I,|I| \leqslant m$, of uniform covers, there exists a uniform cover $\mathscr{Q}$ which refines each $\mathcal{U}_{i}$. $\mathcal{Q}(m)$ will denote the class of all uniform spaces which admit $m$. It is easily verified that $\mathscr{Q}(m)$ is a coreflective subcategory of Unif.

Finally, define $\mathscr{B}(m)$ to be the class of all uniform spaces $X$ such that $X$ has covering character at most $m$ and admits all cardinals less than $m$.

For a reference point, we remark that $\mathscr{B}\left(\boldsymbol{\aleph}_{0}\right)$ is the class of all precompact spaces. If $m$ is a singular cardinal, $\mathscr{B}(m)$ consists of the discrete spaces of power less than $m$. If $m$ is a regular cardinal, there exist nondiscrete members of $\Re(m)$ : let $|X|=m$ and define $\mathcal{Q}$ to be a uniform cover if there exists $U$ in $\mathcal{Q}$ such that $|X-U|<m$. One may show that every class $\mathscr{B}(m) \subset \Lambda$ and that $\mathscr{B}(k) \cap \Re(m)$ contains only discrete spaces if $k \neq m$.

Let $\lambda$ : Unif $\rightarrow \Lambda$ be the locally fine coreflection operator. We note the following facts about locally fine spaces:

(i) For each uniform space $X$, the identity mapping $\lambda(X \times X) \rightarrow X * X$ is uniformly continuous [5, III.28].

(ii) If $X$ is locally fine, the diagonal mapping $\Delta: X \rightarrow X * X$ is uniformly continuous. (This follows from (i) since $\Delta: X \rightarrow \lambda(X \times X)$ is uniformly continuous.)

(iii) If $\delta \subset \Lambda$, then $\delta$ satisfies Axiom II. (Assume that $\hat{f}: S \rightarrow U(S, Y)$ is uniformly continuous. Then $f: S * S \rightarrow Y$ is uniformly continuous, so by (ii), $f_{\Delta}=f \circ \Delta: S \rightarrow Y$ is uniformly continuous.)

It follows from Theorem 1 that if $\mathcal{S}$ is a finitely productive subfamily of $\Lambda$, then $\bigodot$ is cartesian-closed.

EXAmples. (1) For any $m \geqslant \aleph_{0}, \operatorname{co}(\Re(m))$ is cartesian-closed since $\mathscr{B}(m)$ is a finitely productive subfamily of $\Lambda$. 
(2) Let $I$ denote the closed unit interval with the usual metric uniformity. Then $e_{1}=\operatorname{co}(\{I\})$ is cartesian-closed since $I \times I$ is inductively generated from $I$. For essentially the same reason, each of the following classes are cartesian-closed.

(3) $e_{2}=\operatorname{co}\left(\left\{2^{\aleph_{0}}\right\}\right)(=\operatorname{co}\{$ compact metric spaces $\}=\operatorname{co}(S)$, where $S=\{0\} \cup$ $\{1 / n: n=1,2, \ldots\})$. We note that $C_{2}$ is the class of fine spaces which have sequential topologies.

(4) $e_{3}=\operatorname{co}\{$ precompact metric spaces $\} \quad\left(=\operatorname{co}\left(S^{\prime}\right)\right.$, where $S^{\prime}=\{1 / n: n=$ $1,2, \ldots\})$.

We remark that $\mathcal{C}_{1} \subsetneq C_{2} \subsetneq C_{3}$ since $2^{\aleph_{0}} \notin \operatorname{co}(\{I\})$ and the open unit interval is not a member of $e_{2}$ (since it is not fine).

The remainder of the paper will be devoted to showing that any subfamily $\delta$ of Unif which satisfies the conditions of Theorem 1 is contained in a finitely productive family $\mathcal{S}^{\prime} \subset \Lambda \cap \mathcal{C}$ (Corollary 2 ).

Proposition 5. Assume that $\mathcal{S}$ satisfies Axiom $\mathrm{I}$ and $\mathcal{C}$ is cartesian-closed. If $\mathcal{S}$ contains an infinite space $S$ which admits $\aleph_{0}$, then either $\delta \subset \mathcal{D}$ or $\delta \subset \mathscr{B}(m)$ for some $m \geqslant \boldsymbol{\aleph}_{1}$.

Proof. Without loss of generality, we may assume that $\delta$ is closed under the formation of quotients since the collection of all quotients of members of $\delta$ also satisfies Axiom I (see [5, III, Exercise 8]).

Claim 1. If $\delta$ contains a space $X$ which admits $\boldsymbol{\aleph}_{0}$ and has covering character $m \geqslant \boldsymbol{\kappa}_{1}$, then all discrete spaces of power less than $m$ belong to $\delta$. To prove this, assume $k<m$ and choose a metric space $M_{k}$ with covering character $\geqslant k$ and an onto uniformly continuous mapping $f: X \rightarrow M_{k}$. Let $d M_{k}$ denote the discrete coreflection of $M_{k}$. Since $X$ admits $\boldsymbol{\aleph}_{0}$ and $M_{k}$ has a countable uniform base, $f$ : $X \rightarrow d M_{k}$ is uniformly continuous, so $f$ is a quotient mapping and $d M_{k}$ is a member of $\delta$. Since $\left|M_{k}\right| \geqslant k$, the claim is established.

The space $S$ guaranteed by assumption has covering character $\geqslant \aleph_{1}$, since no infinite precompact space admits $\aleph_{0}$. Hence by Claim 1 , the countably infinite discrete space $\mathbf{N}$ is a member of $\mathcal{S}$.

ClaIM 2. If the discrete space $D$ is a member of $\mathcal{S}$, then every member of $\mathcal{S}$ admits $|D|$. Let $m=|D|$ and assume $X$ is a member of $\delta$. By Axiom I, $X \otimes D=X \times D$. Since $C$ is cartesian-closed, the product $\otimes$ preserves sums; hence $X \times D$ is uniformly isomorphic to the sum $\sum_{i \in D} X_{i}\left(X_{i}=X\right)$, which implies that $X$ admits $m$.

In particular, every member of $S$ admits $\aleph_{0}$ since $\mathbf{N}$ is a member of $S$.

ClaIm 3. If $\delta$ contains a space $X$ with covering character $m$, then every member of $\delta$ admits $k$, for each $k<m$. Since $m \geqslant \aleph_{1}$ and $k<m$, Claim 1 implies that the discrete space of power $k$ belongs to $\mathcal{S}$. Then Claim 2 implies that every member of $\mathcal{S}$ admits $k$.

Now consider the class of cardinals $\{k: \delta \cap \mathcal{C}(k) \neq \varnothing\}$. If this class has a supremum $m$ and $k<m$, then $\delta$ contains a space with covering character $>k$, so by Claim 3 every member of $\delta$ admits $k$. It follows that $\delta \subset \mathscr{B}(m)$ and $m \geqslant \boldsymbol{\aleph}_{1}$. If this class has no supremum, Claim 3 implies that every member of $\delta$ admits all cardinals, so $\delta \subset \mathscr{Q}$. 
For our next proposition, we will need the following lemma.

LEMma 1. Assume that $C$ is a cartesian-closed coreflective subcategory of Unif. Define $\mathfrak{S}_{Y}=\{X \in \mathcal{C}$ : the identity mapping $c U(X, Y) \rightarrow[X, Y]$ is uniformly continuous $\}, Y$ in $\mathcal{C}$. Then every class $\mathcal{S}_{Y}$ is closed under the formation of quotients.

Proof. Let $S$ be a member of $\mathcal{S}_{Y}$ and let $q: S \rightarrow T$ be a quotient mapping. We will show that the evaluation mapping $e_{T}: c U(T, Y) \otimes T \rightarrow Y$ is uniformly continuous; then by definition, $T$ will be a member of $\delta_{Y}$. By assumption, the evaluation mapping $e_{S}: c U(S, Y) \otimes S \rightarrow Y$ is uniformly continuous and the mapping [q]: $c U(T, Y) \rightarrow c U(S, Y)$ is uniformly continuous. Define $\hat{e}: c U(T, Y) \otimes S \rightarrow Y$ by $\hat{e}=e_{T} \circ(i \times q)$, where $i$ is the identity mapping on $U(T, Y)$, and observe that $\hat{e}(f, s)=f(q(s))$. It follows that $\hat{e}=e_{S} \circ([q] \times j)$, where $j$ is the identity mapping on $S$, so $\hat{e}$ is unformly continuous. Since $e$ is cartesian-closed, the product $\otimes$ preserves quotient mappings, so $i \times q$ is a quotient mapping and therefore $e_{T}$ is uniformly continuous.

Proposition 6. Assume that $\delta$ satisfies Axiom I and $\mathcal{C}$ is cartesian-closed. Assume that $I=[0,1]$ is a member of $C$ and that the identity mapping $i: c U(S, I) \rightarrow[S, I]$ is uniformly continuous for all $S$ in $\delta$. Then $\delta \subset \Re\left(\boldsymbol{\aleph}_{0}\right)$.

Proof. In the language of Lemma 1 , we are assuming that $\delta \subset \delta_{I}$; hence by Lemma 1 we may assume that $\delta$ is closed under the formation of quotients.

Assume that $\delta$ contains a nonprecompact space $S$. Then there exists a nonprecompact metric space $M$ and an onto uniformly continuous mapping $q: S \rightarrow M$. Let $N=\left\{x_{n}: n=1,2, \ldots\right\}$ be an infinite uniformly discrete subspace of $M$.

Let $B(n, k)$ be the sphere in $M$ with center $x_{n}$ and radius $\frac{1}{k}$, and define $\mathcal{U}(n, k)=\left\{B(n, k), M-\left\{x_{n}\right\}\right\}$. Each $\mathcal{Q}(n, k)$ is a two-element uniform cover of $M$. Let $\mathcal{V}$ be the uniform cover of $I$ consisting of spheres of radius $\frac{1}{2}$. For each pair $(n, k)$, there exists a uniformly continuous mapping $f_{n, k}: M \rightarrow I$ such that $f_{n, k}^{-1}(\mathscr{V})<$ $\mathcal{Q}(n, k)$ (for example, if $d$ is the metric on $M$, define $f_{n, k}(x)=k d(x, X-B(n, k)$ ) $\wedge 1$ ). Re-index the above families as $\left\{f_{m}\right\}$ and $\left\{\mathscr{U}_{m}\right\}$ respectively, $m=1,2, \ldots$, and define $\hat{h}: N \rightarrow U(M, I)$ by $\hat{h}\left(x_{m}\right)=f_{m}$. Let $q M$ be the quotient space induced by the mapping $q$. Then $N$ is a discrete subspace of $q M$ and $q M$ is a member of $\delta$.

By [5, III.18], $U(M, I)$ is an injective uniform space, so $\hat{h}$ may be extended to a uniformly continuous mapping $\hat{\phi}: q M \rightarrow U(M, I)$. Since $U(M, I)$ is a subspace of $U(q M, I)$ and $i: c U(q M, I) \rightarrow[q M, I]$ is uniformly continuous, it follows that $\hat{\phi}$ : $q M \rightarrow[q M, I]$ is uniformly continuous. Let $\phi: q M \otimes q M \rightarrow I$ be the mapping associated with $\hat{\phi}$. Since $\delta$ satisfies Axiom I, $q M \otimes q M=q M \times q M$. Therefore the family $\{\phi(x,-): q M \rightarrow I\}$ is equi-uniformly continuous. Choose a uniform cover $\mathscr{Q}$ of $q M$ such that $\mathscr{Q}<f_{m}^{-1}(\mathfrak{V})$ for $m=1,2, \ldots$ (noting that $f_{m}=\phi\left(x_{m},-\right)$ ). Since $\mathscr{Q}<\mathscr{Q}_{m}, m=1,2, \ldots, \mathscr{Q}$ refines the countable partition $\left\{M-\left\{x_{1}, x_{2}, \ldots\right\}\right\} \cup$ $\left\{\left\{x_{m}\right\}: m=1,2, \ldots\right\}$; hence $N$ is a quotient of $q M$ and therefore a member of $\delta$.

By Proposition 5, $N$ a member of $\delta$ implies that every member of $\mathcal{C}$ admits $\boldsymbol{\aleph}_{0}$, contradicting the fact that $I$ is a member of $\mathcal{C}$. Hence we have established that every member of $\delta$ is precompact. 
REMARK. The preceding proposition and Theorem 1 show that if $\delta$ satisfies Axioms $I$ and II and $[0,1]$ is a member of $\mathcal{C}$, then every member of $\delta$ is precompact. To treat the case when $I$ is not a member of $\mathcal{C}$, we will need the following concepts.

A zero set in a uniform space $X$ is a set of the form $f^{-1}\{0\}$, where $f$ is a member of $U(X, I)$. A Baire set in $X$ is a member of the $\sigma$-field generated by the family of zero sets. The symbol Ba-fine $\left.\right|_{I}$ will denote the class of all uniform spaces $X$ such that every finite partition of $X$ into Baire sets is a uniform cover. This class is a coreflective subcategory of Unif (see [1 or 3]). We will need the following result from [11].

TheOREM A. Assume that $\mathcal{C}$ is a coreflective subcategory of Unif. The following statements are equivalent:

(1) $I=[0,1]$ is not a member of $\bigodot$.

(2) $\circlearrowright \subset$ Ba-fine $\left.\right|_{I}$.

(3) Every metric space belonging to $\mathcal{C}$ is discrete.

LemMa 2. Assume that $\mathcal{S}$ satisfies Axiom $\mathrm{I}$ and $\mathcal{C}=\operatorname{co}(\mathcal{S})$. If $\mathrm{I}$ is not a member of $\mathcal{\complement}$, then $\mathcal{C} \mathbb{Q}\left(\boldsymbol{\aleph}_{0}\right)$.

Proof. Since $\mathcal{Q}\left(\boldsymbol{\aleph}_{0}\right)$ is a coreflective subcategory, it suffices to show that $\delta \subset$ $Q\left(\boldsymbol{N}_{0}\right)$.

Let $S$ be a member of $\delta$. By Axiom I, $S \times S$ is a member of $\mathcal{C}$. To show that $S$ admits $\boldsymbol{\aleph}_{0}$, let $\left\{\mathscr{U}_{m}: m=1,2, \ldots\right\}$ be a sequence of uniform covers of $S$. Choose a uniformly continuous pseudometric $d$ on $S$ (bounded by 1) such that the cover $\mathcal{V}$ consisting of the $d$-spheres of radius zero refines each $\mathcal{U}_{n}$. We will show that $\mathscr{V}$ is a uniform cover of $S$.

Since $d: S \times S \rightarrow I$ is uniformly continuous, $\mathscr{Q}=\left\{d^{-1}(0), d^{-1}(0,1]\right\}$ is a finite Baire partition of $S \times S$; hence by Theorem A, $\mathcal{Q}$ is a uniform cover of $S \times S$, so there exists a uniform cover $\mathscr{W}$ of $S$ such that $\mathscr{W} \times \mathcal{W} \cup$. Clearly, for each $W$ in W, $W \times W \subset d^{-1}(0)$; hence $W<\mathcal{V}$ implies that $\mathfrak{V}$ is a uniform cover.

THEOREM 2. The following statements are equivalent to conditions (1)-(5) of Theorem 1:

(6) $\delta$ satisfies Axiom I and either $\delta \subset \mathscr{D}$ or $\delta \subset \mathscr{B}(m)$, for some $m \geqslant \boldsymbol{\aleph}_{0}$.

(7) $\S$ satisfies Axiom I and $\subseteq \subset \Lambda$.

(8) $S * T=S \otimes T$ for all $S$ and $T$ in $\delta$.

Proof. We will establish the following implications: $(5) \rightarrow(6) \rightarrow(7) \rightarrow(8) \rightarrow(5)$.

$(8) \rightarrow(5)$ is immediate.

(6) $\rightarrow(7)$ is immediate since $\mathscr{D} \cup \Re(m) \subset \Lambda$ for all $m \geqslant \boldsymbol{\aleph}_{0}$.

$(5) \rightarrow(6)$. There are two cases. If $I$ is a member of $\mathcal{C}$, then $\mathcal{S} \subset \mathscr{B}\left(\boldsymbol{\aleph}_{0}\right)$ by Proposition 6. If $I$ is not a member of $\mathcal{C}$, then every member of $\mathcal{C}$ admits $\boldsymbol{\kappa}_{0}$ by Lemma 2. If every member of $\delta$ is finite, then $\delta \subset \mathscr{D}$; otherwise, $\delta$ contains an infinite space which admits $\aleph_{0}$, so by Proposition 5 either $\delta \subset \mathscr{D}$ or $\delta \subset \Re(m)$, for some $m \geqslant \aleph_{1}$. 
(7) $\rightarrow$ (8). Since $\Lambda$ is a coreflective subcategory, $\subset \subset \Lambda$. Then by Axiom I, $S \times T$ is locally fine for all $S$ and $T$ in $\delta$. In general, the identity mappings $\lambda(S \times T) \rightarrow S * T$ $\rightarrow S \otimes T$ are uniformly continuous, so $S * T=S \times T=S \otimes T$.

REMARK. The proof given above shows that if $\mathcal{C}=\operatorname{co}(\delta)$ satisfies the conditions of Theorems 1 and 2, then

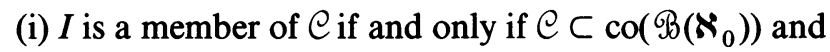

(ii) $I$ is not a member of $\mathcal{C}$ if and only if $\mathcal{C} \subset \mathcal{Q}\left(\boldsymbol{\aleph}_{0}\right)$.

As a corollary to the above theorem, we can now show that $\mathcal{C}=\operatorname{co}(\mathcal{S})$ satisfies the conditions of Theorems 1 and 2 if and only if $\mathcal{C}$ is inductively generated by a finitely productive subfamily of $\Lambda$.

Corollary 2. Assume that $\mathcal{C}=\operatorname{co}(\delta) \neq \mathscr{D}$. The following statements are equivalent:

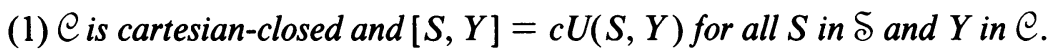

(2) There exists a finitely productive subfamily $\mathcal{S}^{\prime} \subset \Re(m)$, for some $m \geqslant \aleph_{0}$, such that $\delta \subset \mathcal{S}^{\prime} \subset \mathcal{C}$.

(3) There exists a finitely productive subfamily $\delta^{\prime} \subset \Lambda$ such that $\delta \subset \delta^{\prime} \subset \circlearrowright$.

Proof. (2) $\rightarrow(3)$ is immediate.

$(3) \rightarrow(1)$. $\delta^{\prime}$ satisfies Axiom I since it is finitely productive, so (1) follows from Theorems 1 and 2.

$(1) \rightarrow(2)$. Define $\mathcal{S}^{\prime}=\{X \in C:[X, Y]=c U(X, Y)$ for all $Y$ in $\mathcal{C}\}$. Then $\delta \subset \mathcal{S}^{\prime}$

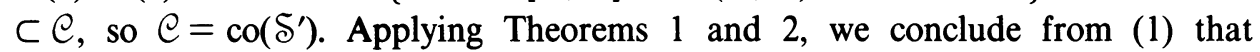
$\delta^{\prime} \subset \mathscr{B}(m)$, for some $m \geqslant \boldsymbol{\aleph}_{0}$. We will now show that $\mathcal{S}^{\prime}$ is finitely productive.

By Theorem 1, $\mathcal{S}^{\prime}$ satisfies Axiom I, so $S \times T$ is a member of $\mathcal{C}$ for all $S$ and $T$ in $\mathcal{S}^{\prime}$. We must show that for all $Y$ in $\mathcal{C},[S \times T, Y]=c U(S \times T, Y)$.

By Theorem $1,[S \times T, Y]=c U_{\S}(S \times T, Y)$. Since the identity mapping $i: U(X, Y) \rightarrow U_{\delta}(X, Y)$ is uniformly continuous for all $X$ in $\mathcal{C}$, it follows that $i: c U(S \times T, Y) \rightarrow[S \times T, Y]$ is uniformly continuous.

Let $\phi:[S \times T, Y] \rightarrow[T,[S, Y]]$ be the natural bijection. Since $\mathcal{C}$ is cartesian-closed, $\phi$ is a uniform isomorphism. Let $\hat{\mathcal{U}}$ be the basic uniform cover of $U(S \times T, Y)$ induced by the uniform cover $\mathcal{Q}$ of $Y$. Choose a uniform cover $\mathscr{V}$ such that $\mathcal{V}^{*}<\mathcal{Q}$. $\mathcal{V}$ induces a uniform cover $\mathcal{V}_{S}$ of $U(S, Y)$. Since $S$ is a member of $\mathcal{S}^{\prime}, \mathcal{V}_{S}$ is also a uniform cover of $[S, Y]$, so it induces a uniform cover $\mathcal{V}_{S T}$ of $U(T,[S, Y])$. Since $T$ is a member of $\mathcal{S}^{\prime}, \mathfrak{V}_{S T}$ is also a uniform cover of $[T,[S, Y]]$. Using the naturality of $\phi$, one may show that $\phi^{-1}\left(\mathcal{V}_{S T}\right)<\hat{\vartheta}$ (see the proof of Theorem 1 in [10]); hence $\hat{\vartheta}$ is a uniform cover of $[S \times T, Y]$, so $i:[S \times T, Y] \rightarrow c U(S \times T, Y)$ is uniformly continuous.

The preceding paragraphs establish that $[S \times T, Y]=c U(S \times T, Y)$, so the proof of Corollary 2 is complete.

\section{REFERENCES}

1. Z. Frolik, Measurable uniform spaces, Pacific J. Math. 55 (1974), 93-105.

2. S. Ginsburg and J. Isbell, Some operators on uniform spaces, Trans. Amer. Math. Soc. 93 (1959), $145-168$. 
3. A. W. Hager, Three classes of uniform spaces, Proc. Third Prague Topological Sympos., Academia, Prague, 1972, pp. 159-164.

4. H. Herrlich, Cartesian-closed topological categories, Math. Colloq. Univ. Cape Town 9 (1974), 1-15.

5. J. Isbell, Uniform spaces, Amer. Math. Soc., Providence, R.I., 1964.

6. J. F. Kennison, Reflective functors in general topology and elsewhere, Trans. Amer. Math. Soc. 118 (1965), 303-315.

7. S. Mac Lane, Categories for the working mathematician, Springer-Verlag, New York, 1971.

8. L. D. Nel, Cartesian-closed coreflective hulls, Quaestiones Math. 2 (1977), 269-283.

9. G. Tashjian, Cartesian-closed coreflective subcategories of Unif., Proc. Fourth Prague Topological Sympos., 1976, Part B, Polygrafia, Prague, 1977, pp. 454-460.

10. __ Cartesian-closed coreflective subcategories of Tychonoff spaces, Pacific J. Math. 81 (1979), $547-558$.

11. G. Tashjian and J. Vilimovský, Coreflective not preserving the interval, and Baire partitions of uniform spaces, Proc. Amer. Math. Soc. 77 (1979), 257-263.

Department of Mathematics, George Mason University, Fairfax, Virginia 22030

Department of Mathematics, Merrimack College, North Andover, Massachusetts 01845 